\title{
Elemente der Planeten (313), (318), (319) und (320).
}

Von $A$. Berberich.

\section{Planet (313) Chaldaea.*)}

Die Elemente sind aus 3 Beobachtungen 1891 Aug. 30 , Sept. 24 und Oct. ro abgeleitet.

Epoche r8gr Oct. 10.5 M. Z. Berlin.

$$
\begin{aligned}
& M=238^{\circ} 2 \mathrm{x}^{\prime} 5^{\prime \prime} .4 \\
& \omega=31120 \quad 10.7 \\
& \delta=176 \text { 41 } 33.7 \text { Aequ. } 1891.0 \\
& i=\begin{array}{llll}
1 & 28 & 36.4
\end{array} \\
& \varphi=1017 \quad 15.7 \\
& \mu=966.584 \\
& \log a=0.37651 \mathrm{x}
\end{aligned}
$$

Der Planet hatte zur Zeit der Entdeckung die Grösse I I⿳⺈.⺕o. In der nächsten Opposition 1893 März dürfte er ungefähr anderthalb Grössen heller werden.

\section{Planet (318) (Charlois 1891 Sept. 24).}

Die Beobachtungen Nizza Sept. 24, Oct. 9 und Wien Oct. 2 I haben folgende Elemente ergeben:

Epoche 1891 Oct. 2 I.5 M. Z. Berlin.

$$
\begin{aligned}
& M=284^{\circ} 38^{\prime} 5^{\prime \prime} \text {.。 } \\
& \omega=260 \quad 18 \quad 23.5 \\
& \Omega=16259 \text { 0.5 Aequ. 1891.0 } \\
& i=\begin{array}{lll}
10 & 26 & 10.7
\end{array} \\
& \varphi=6136.3 \\
& \mu=597.919 \\
& \log _{9} a=0.515576
\end{aligned}
$$

Auch bei diesem Planeten werden in der nächsten Opposition, I892 November, die Helligkeitsverhältnisse günstiger sein.

Bemerkenswerth ist die Commensurabilität der mittleren Bewegung mit der des Jupiter; weitere Beobachtungen werden entscheiden lassen, ob dieselbe bestehen bleibt.

\section{Planet (319) (Charlois 1891 0ct. 8).}

Bisher ist nur eine Kreisbahn $\left(\mu=577^{\prime \prime}\right)$ aus Oct. 8 und ro abgeleitet worden. Eine Wiener Beobachtung vom 24. Oct. weicht um $4^{\text {s }}$ von dem Ephemeridenorte ab.

\section{Planet (320) (Palisa 1891 0ct. 11).}

Aus Beobachtungen von Oct. I I bis Nov. 3 sind folgende Elemente abgeleitet:

$$
\begin{aligned}
& \text { Epoche } 189 \mathrm{I} \text { Nov. 3.5 M. Z. Berlin. } \\
& M=9^{\circ} 3 x^{\circ} 43^{\prime \prime} \circ \\
& \omega=153 \text { I } 54.8 \\
& \delta=22135 \quad 59.7 \quad \text { Aequ. } 1891.0 \\
& i=85438.5 \\
& \varphi=7133^{8.7} \\
& \mu=682: 679 \\
& \log a=0.477193
\end{aligned}
$$

Wegen der kurzen Zwischenzeit dürten die Elemente noch etwas unsicher sein.

\section{Berlin 189 × Nov. I1. A. Berberich} Hullfsarbeiter am Kgl. Recheninstitut.

*) Nach Gould's Astronomical Journal Nr. 248 hat der Planet (313) (Palisa 1891 Aug. 30) den Namen Chaldaea erhalten. Kr.

\section{Weitere Notizen zur totalen Mondfinsterniss 1891 Nov, 15.}

Nach ferneren hier eingegangenen Nachrichten scheint das Wetter leider fast überall ungünstig gewesen zu sein. Totales Misslingen wird gemeldet aus Basel, Christiania, Genf, Karlsruhe, Kremsmünster, Upsala, Zürich.

In Berlin, Urania konnten der erste Contact und eine Reihe Kraterbedeckungen sowie 4 Sternaustritte beobachtet werden.

In Bonn hat Dr. Lenard das Ende der Totalität sowie eine Anzahl Kraterbedeckungen beobachtet.

In Düsseldorf wurde nur der Anfang der Finsterniss notirt.

In Göttingen gelangen Kraterbedeckungen, sowie einige Sternbedeckungen.

In Jena sind Krater-Ein- und -Austritte beobachtet, die Bedeckungen der Sterne waren nicht wahrzunehmen.

In Kalocsa wurden nur einige Kraterbedeckungen notirt.

In Prag gelangen Beobachtungen von Kraterbedeckungen.

In Turin ebenso.

Verkanfs-Anzeige. Im Auftrage habe ich zu verkaufen die Bibliothek eines der hervorragendsten Astronomen. Sie ist von bedeutendem Werthe und enthält bei ihrer Reichhaltigkeit Alles, dessen eine Sternwarte bedarf, um in allen Theilen der Astronomie speciell der praktischen mit reichen literarischen Hülfsmitteln vollständig ausgerüstet zu sein. Reflectanten werden gebeten sich mit mir in Verbindung zu setzen.

$$
\text { Voss' Sortiment (G. Haessel) in Leipzig. }
$$

In halt zu Nr. 3067. W. F. Wislecinus. Ueber den Einfluss von Ring- und Scheibenblenden auf Mikrometermessungen. 345. - Eschenhagen. Das Erdbeben in Japan am 27. October 1891. 357. - W. Luther. Beobachtungen des Planeten (313) Chaldaea. 357. A. Berberich. Elemente der Planeten (3 3 ), (318), (319) und (320). 359. - Weitere Notizen zur totalen Mondfinsterniss I891 Nov. I 5. 359. - Verkaufsanzeige. 359 . 